Pacific Journal of Mathematics

INCREASING SEQUENCES OF ET N 


\section{INCREASING SEQUENCES OF BETTI NUMBERS}

Eugene H. Gover and Mark Ramras

We study the sequence of Betti numbers $\left\{\beta_{i}(M)\right\}_{i \geq 1}$ of an arbitrary finitely generated nonfree module $M$ over a commutative noetherian local ring $R$ and show that for a certain class of rings this sequence is always nondecreasing, while for a certain subclass of rings, the subsequence $\left\{\beta_{i}(M)\right\}_{i \gtrless 2}$ is strictly increasing.

In [3], a class of commutative noetherian local rings $(R, \mathfrak{m})$ called BNSI rings was introduced. These rings have the property that for every finitely generated nonfree module $M$, the sequence of Betti numbers $\left\{\beta_{i}(M)\right\}_{i \geqq 1}$ is strictly increasing. Recall that $\beta_{i}(M)$ is the dimension of the $R / \mathfrak{m}$-vector space $\operatorname{Tor}_{i}^{i R}(M, R / \mathfrak{m})$; equivalently, it is the rank of the free module $F_{i}$ where

$$
\cdots \longrightarrow F_{i} \longrightarrow F_{i-1} \longrightarrow \cdots \longrightarrow F_{0} \longrightarrow M \longrightarrow 0
$$

is a minimal $R$-free resolution of $M$. A class of BNSI rings was given in [3, Theorem 3.2A]: Let $(S, \mathfrak{n})$ be a noetherian local ring and let $J$ be an ideal which is not contained in any prime ideal of grade 1 . If $S$ is a domain, then $S / \mathfrak{n} J$ is a BNSI ring.

In this note, using a result of G. Levin [2] we prove:

THEOREM 1.1. Let $(S, \mathfrak{n})$ be a noetherian local ring of Krull dimension $d \geqq 2$. Then for $n$ sufficiently large, the local ring $(R, \mathfrak{m})=\left(S / \mathfrak{n}^{n}, \mathfrak{n} / \mathfrak{n}^{n}\right)$ has the property that for all finitely generated nonfree $R$-modules $M$, the sequence $\left\{\beta_{i}(M)\right\}_{i \geqq 2}$ is strictly increasing. In fact, for all $i \geqq 2, \beta_{i+1}(M)-\beta_{i}(M) \geqq d-1$.

Thus $R$ is nearly a BNSI ring, except that our proof gives no estimate for $\beta_{2}(M)-\beta_{1}(M)$. Another drawback is that we can not estimate how large $n$ must be, since it comes, indirectly, from the Artin-Rees Lemma. To fill these gaps (at least partially) we offer the weaker, but more general:

CoROllary 2.2. Let $(S, \mathfrak{n})$ be a noetherian local ring of Krull dimension $\geqq 1$, and let $R=S / \mathfrak{n}^{n}$, with $n \geqq 1$. Then for all finitely generated $R$-modules $M$, the sequence $\left\{\beta_{i}(M)\right\}_{i \geqq 1}$ is nondecreasing.

It should be pointed out that if $S$ is assumed to be a domain and grade $\mathfrak{n} \geqq 2$, then by the theorem from [3] cited above, $S / \mathfrak{n}^{n}$ is a BNSI ring for all $n \geqq 2$. 
1. We begin with:

Proof of Theorem 1.1. Let $0 \rightarrow K \rightarrow R^{n_{0}} \rightarrow M \rightarrow 0$ be exact, with $K \subset \mathfrak{m} R^{n_{0}}$, and let

$$
\cdots \longrightarrow R^{n_{i}} \longrightarrow \cdots \longrightarrow R^{n_{1}} \longrightarrow K \longrightarrow 0
$$

be a minimal $R$-free resolution of $K$. Then $n_{i+1}=\beta_{i}(K)=\beta_{i+1}(M)$, and since $K \subset m R^{n_{0}}, \operatorname{ann}(m) \cdot K=0$. Similarly, all the higher syzygies of $M$ are annihilated by ann(m). Thus it suffices to prove that for any finitely generated $R$-module $N$ which is annihilated by ann(nt), $\beta_{2}(N)-\beta_{1}(N) \geqq d-1$.

By [2, Formula (8), p. 9], for $n$ sufficiently large we have

$$
P_{R}^{N}(t)=P_{S}^{N}(t) / 1-t\left(P_{S}^{R}(t)-1\right)
$$

where for any noetherian local ring $Q$ and finitely generated $Q$ module $X, P_{Q}^{X}(t)$ is the Poincaré series $\sum_{n=0}^{\infty} \beta_{i}(X) t^{i}$. Now $P_{S}^{\prime}(t)=$ $1+b_{2} t+\cdots$. Since

$$
S^{b_{2}} \longrightarrow S \longrightarrow R \longrightarrow 0
$$

is part of a minimal S-resolution of $R, b_{2}=$ the minimal number of generators of $\mathfrak{n t}^{n}$. But $\mathfrak{n}^{n}$ is $\mathfrak{t}$-primary, and so by Krull's Generalized Ideal Theorem [1, Theorem 152], $b_{2} \geqq$ height $\mathfrak{\imath}=d$. Now

$$
1-t\left(P_{S}^{R}(t)-1\right)=1-b_{2} t^{2}-\cdots
$$

and so if $\left(1-b_{2} t^{2}-\cdots\right)^{-1}=\sum_{i=0} c_{i} t^{i}$, then $c_{0}=1, c_{1}=0$, and $c_{2}=$ $c_{0} b_{2}=b_{2}$.

Now let $P_{S}^{N}(t)=\sum_{j=0}^{\infty} p_{j} t^{j}$. Thus

$$
S^{p_{2}} \longrightarrow S^{p_{1}} \longrightarrow S^{p_{0}} \longrightarrow N \longrightarrow 0
$$

is part of a minimal $S$-free resolution of $N$. We claim that $p_{2}+p_{0} \geqq$ $p_{1}$. To see this, localize at a minimal prime of $S$ to obtain an artin ring $T$. Then the sequence

$$
T^{p_{2}} \stackrel{f}{\longrightarrow} T^{p_{1}} \stackrel{g}{\longrightarrow} T^{p_{0}}
$$

is exact, so $l\left(T^{p_{1}}\right)=l(\mathrm{im} f)+l(\mathrm{im} g) \leqq l\left(T^{p_{2}}\right)+l\left(T^{p_{0}}\right)$, where $l(X)$ denotes the length of $X$. Therefore $p_{1} \leqq p_{2}+p_{0}$. Now from $\left({ }^{*}\right)$ we have

$$
P_{R}^{N}(t)=\left(\sum_{i=1}^{\infty} c_{i} t^{i}\right)\left(\sum_{j=0}^{\infty} p_{j} t^{j}\right)=\sum_{k=0}^{\infty} \beta_{k} t^{k} .
$$

Thus $\beta_{1}=c_{0} p_{1}+c_{1} p_{0}=p_{1}$, and $\beta_{2}=c_{0} p_{2}+c_{1} p_{1}+c_{2} p_{0}=p_{2}+b_{2} p_{0}$. Since $\quad b_{2} \geqq d \geqq 2, \beta_{2} \geqq p_{2}+p_{0}+(d-1) p_{0} \geqq p_{1}+(d-1) p_{0}=\beta_{1}+$ 
$(d-1) p_{0}$. So $\beta_{2}-\beta_{1} \geqq(d-1) p_{0} \geqq d-1 \geqq 1$.

2. We now remove the restriction that $n$ be "sufficiently large". Our starting point is [3, Theorem 3.4]: Let $(S, \mathfrak{n})$ be a noetherian local domain and let $J$ be any nonzero ideal. Let $R=S / \mathfrak{n} J$. Then for any finitely generated $R$-module $M$, the sequence $\left\{\beta_{i}(M)\right\}_{i \unlhd 1}$ is nondecreasing.

The proof of this result was a minor modification of the proof of [3, Theorem 3.2]. A further modification yields:

Proposition 2.1. Let $(S, \mathfrak{n})$ be a noetherian local ring and let $J$ be a nonnilpotent ideal. Let $R=S / \mathfrak{n} J$. Then for any finitely generated $R$-module $M$, the sequence $\left\{\beta_{i}(M)\right\}_{i \geq 1}$ is nondecreasing.

Proof. Following the proof of [3, Theorem 3.4] we obtain an $S$-module $A$ such that $J S^{p} \subset A \subset S^{p}$, where $p=\beta_{1}(M)$, and $\beta_{2}(M)=$ the minimal number of generators of $A$. Thus we must show that $A$ can not be generated by $p-1$ elements.

Let $x \in J$ be a nonnilpotent element, and let $T$ be the localization of $S$ at the multiplicative set $\left\{x^{i} \mid i \geqq 0\right\}$. Then

$$
J S^{p} \bigotimes_{S} T \subset A \bigotimes_{S} T \subset S^{p} \bigotimes_{S} T=T^{p} .
$$

Since $J$ meets the multiplicative set, $J S^{p} \bigotimes_{S} T=T^{p}$. Hence $A \bigotimes_{S}$ $T=T^{p}$. Now the minimal number of generators of $A$ as an $S$ module is at least the minimal number of generators of $A \otimes_{S} T$ as a $T$-module, and since a free module of rank $p$ can not be generated by $p-1$ elements, we are done.

As an easy consequence we have:

CoRollary 2.2. Let $(S, \mathfrak{n})$ be a noetherian local ring of Krull dimension $\geqq 1$, and let $R=S / \mathfrak{n}^{n}$. Then for any finitely generated $R$-module $M$, the sequence $\left\{\beta_{i}(M)\right\}_{i \geqq 1}$ is nondecreasing.

Proof. When $n=1, R$ is a field and all the Betti numbers in the sequence are 0 . For $n \geqq 2$, let $J=\mathfrak{n}^{n-1}$. Since $\operatorname{Krull} \operatorname{dim} S \geqq 1$, $J$ is not nilpotent, and the preceding proposition applies.

\section{REFERENCES}

1. I. Kaplansky, Commutative Rings, Allyn and Bacon, 1970.

2. G. Levin, Poincaré series of modules over local rings, Proc. Amer. Math. Soc., 72 (1978), 6-10.

3. M. Ramras, Betti Numbers and Reflexive Modules, Ring Theory, Academic Press, 1972. 
Received October 18, 1978 and in revised form February 28, 1979. The second author was partially supported by N. S. F. grant MCS 77-03616 during the preparation of this paper.

NORTHEASTERN UNIVERSITY

Boston, MA 02115 


\section{PACIFIC JOURNAL OF MATHEMATICS}

\section{EDITORS}

DONALD BABBITT (Managing Editor)

University of Galifornia

Los Angeles, California 90024

HUGo RossI

University of Utah

Salt Lake City, UT 84112

C. C. MOORE AND ANDREW OGG

University of California

Berkeley, CA 94720

\section{J. DUGUNDJI}

Department of Mathematics University of Southern California Los Angeles, California 90007

R. FINN AND J. MILGRAM Stanford University Stanford, California 94305

ASSOCIATE EDITORS
E. F. BECKENBACH
B. H. NeumanN
F. WoLF
K. YosHIDA

\section{SUPPORTING INSTITUTIONS}

UNIVERSITY OF BRITISH COLUMBIA UNIVERSITY OF SOUTHERN CALIFONIA CALIFORNIA INSTITUTE OF TECHNOLOGY UNIVERSITY OF CALIFORNIA MONTANA STATE UNIVERSITY STANFORD UNIVERSITY UNIVERSITY OF HAWAII UNIVERSITY OF TOKYO UNIVERSITY OF NEVADA, RENO UNIVERSITY OF UTAH NEW MEXICO STATE UNIVERSITY WASHINGTON STATE UNIVERSITY OREGON STATE UNIVERSITY UNIVERSITY OF OREGON UNIVERSITY OF WASHINGTON 


\section{Pacific Journal of Mathematics \\ Vol. 87, No. $1 \quad$ January, 1980}

Spiros Argyros, A decomposition of complete Boolean algebras ..........

Gerald A. Beer, The approximation of upper semicontinuous multifunctions

by step multifunctions . . . . ....................

Ehrhard Behrends and Richard Evans, Multiplicity theory for Boolean

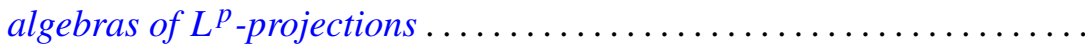

Man-Duen Choi, The full $C^{*}$-algebra of the free group on two

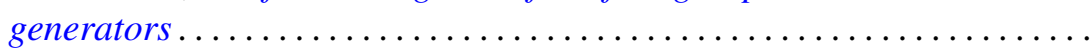

Jen-Chung Chuan, Axioms for closed left ideals in a $C^{*}$-algebra . . . . . . . .

Jo-Ann Deborah Cohen, The strong approximation theorem and locally

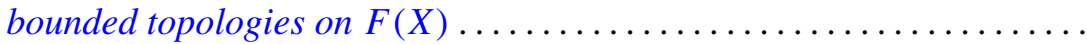

Eugene Harrison Gover and Mark Bernard Ramras, Increasing sequences of Betti numbers............................

Morton Edward Harris, Finite groups having an involution centralizer with

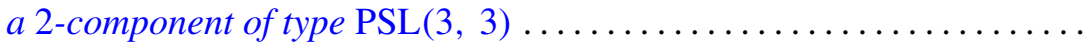

Valéria Botelho de Magalhães Iório, Hopf $C^{*}$-algebras and locally compact

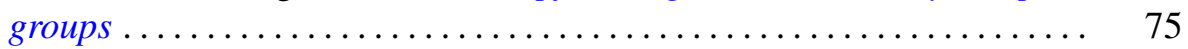

Roy Andrew Johnson, Nearly Borel sets and product measures . . . . . . . . . .

Lowell Edwin Jones, Construction of $Z_{p}$-actions on manifolds . . . . . . . . .

Manuel Lerman and Robert Irving Soare, $d$-simple sets, small sets, and

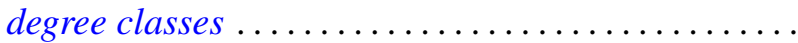

Philip W. McCartney, Neighborly bushes and the Radon-Nikodým property

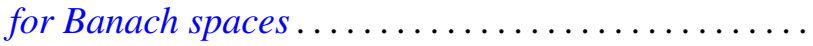

Robert Colman McOwen, Fredholm theory of partial differential equations on complete Riemannian manifolds.

Ernest A. Michael and Carl Preston Pixley, A unified theorem on continuous selections.

Ernest A. Michael, Continuous selections and finite-dimensional sets .

Vassili Nestoridis, Inner functions: noninvariant connected components...

Bun Wong, A maximum principle on Clifford torus and nonexistence of proper holomorphic map from the ball to polydisc.

Steve Wright, Similarity orbits of approximately finite $C^{*}$-algebras . . .

Kenjiro Yanagi, On some fixed point theorems for multivalued

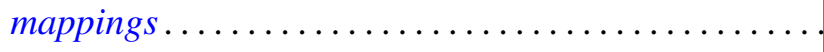

Wieslaw Zelazko, A characterization of LC-nonremovable ideals in commutative Banach algebras 